Original Research Paper

\title{
Image Analysis with Legendre Moment Descriptors
}

\author{
Amy Chiang and Simon Liao \\ Applied Computer Science, The University of Winnipeg, Winnipeg, Manitoba, Canada, R3B 2E9, Canada
}

Received: 23-04-2014

Revised: $12-08-2014$

Accepted: 15-09-2014

Corresponding Author:

Simon Liao

The University of Winnipeg,

Winnipeg, Manitoba, Canada, R3B

2E9, Canada

Email: achiang0602@gmail.com

\begin{abstract}
In this research, a numerical integration method is proposed to improve the computational accuracy of Legendre moments. To clarify the improved computation scheme, image reconstructions from higher order of Legendre moments, up to 240 , are conducted. With the more accurately generated moments, the distributions of image information in a finite set of Legendre moments are investigated. We have concluded that each individual finite set of Legendre moments will represent the unique image features independently, while the even orders of Legendre moments describe most of the image characteristics.
\end{abstract}

Keywords: Legendre Moments, Image Reconstruction, Image Analysis

\section{Introduction}

Moment methods have been the subject of intensive research since the concept of image moments was introduced by (Hu, 1962). Different types of conventional continuous orthogonal moments defined in a rectangular region have been investigated as the unique image shape features for applications in fields of pattern recognition and image analysis. We refer to books written by (Mukundan and Ramakrishnan, 1998; Pawlak, 2006; Flusser et al., 2009) as background study of moment methods for this research.

As one of the important continuous orthogonal moments, the Legendre moment has been well investigated since the earlier years of moment-based descriptors studies (Teague, 1980; Teh and Chin, 1988; Liao and Pawlak, 1996). However, some computational issues have bottlenecked the further development of efficient applications driven by Legendre moment-based techniques. The objective of this research is to study the image representing characteristics of Legendre moments and demonstrate their potential usefulness in the field of image analysis.

In this study, we have analyzed the computational errors and proposed an efficient method to improve the accuracy of Legendre moments computation, especially for the higher order moments. With the substantially improved accurate Legendre moments, the image reconstructions from Legendre moments, up to the order of 240, are performed with highly satisfied results. We have also conducted the image reconstructions from a finite set of Legendre moments. This leads to the clarification that the lower orders of Legendre moments mainly contain fundamental image information, while the higher orders of Legendre moments preserve more detailed image information; especially, the even orders of Legendre moments describe most of the image characteristics.

The organization of this study is as follows. Section 2 will review the general properties of Legendre moments and the computational errors in Legendre moment computing. In section 3, to verify the more accurate Legendre moment computational results, we represent some reconstructed images from the higher orders of Legendre moments. The investigation of representing characteristics of a partial set of Legendre moments in image analysis is performed in section 4. Finally, the concluding remarks are reported in section 5 .

\section{Legendre Moments}

\section{Legendre Polynomials}

The $m$-th order Legendre polynomial (Sansone, 1991) is defined by the Rodrigues formula:

$$
P_{m}(x)=\frac{1}{2^{m} m !} \frac{d^{m}}{d x^{m}}\left(x^{2}-1\right)^{m}
$$

And its recurrent formula is:

$P_{m+1}(x)=\frac{2 m+1}{m+1} x P_{m}(x)-\frac{m}{m+1} P_{m-1}(x)$

The Legendre polynomials $\left\{\mathrm{P}_{\mathrm{m}}(\mathrm{x})\right\}$ are a complete orthogonal basis set on the interval $[-1,1]$ (Courant and Hilbert, 1996): 


$$
\int_{-1}^{1} P_{m}(x) P_{n}(x) d x=\frac{2}{2 n+1} \delta_{m n}
$$

where, $\delta_{m n}$ is the Kronecker symbol.

\section{Legendre Moments}

The $(m, n)$-th order of Legendre moment of an image function $\mathrm{f}(\mathrm{x}, \mathrm{y})$ is defined on the square $[-1,1] \times[-1,1]$ :

$$
\lambda_{m n}=\frac{(2 m+1)(2 n+1)}{4} \int_{-1}^{1} \int_{-1}^{1} f(x, y) P_{m}(x) P_{n}(y) d x d y
$$

where, $\mathrm{m}, \mathrm{n}=0,1,2, \ldots$

In the case of digital image processing, the double integration in (4) needs to be replaced by double summations. Assuming a digital image is sized by $\mathrm{M} \times$ $\mathrm{N}$, (4) becomes:

$$
\lambda_{m n}=\frac{(2 m+1)(2 n+1)}{M N} \sum_{i=1}^{M} \sum_{j=1}^{N} f\left(x_{i}, y_{j}\right) P_{m}\left(x_{i}\right) P_{n}\left(y_{j}\right) \Delta x \Delta y(5)
$$

where, $\Delta x$ and $\Delta y$ are sampling intervals in the $x$ and $y$ directions.

According to the orthogonality property of the Legendre moments, we can reconstruct an original image from an infinite series of its Legendre moments by:

$$
f(x, y)=\sum_{m=0}^{\infty} \sum_{n=0}^{m} \lambda_{m-n, n} P_{m-n}(x) P_{n}(y)
$$

In practice, however, we would have to truncate the infinite series in (6). If only Legendre moments of order
$<M_{\max }$ are given, the original image function $f(x, y)$ can be approximated by a truncated series:

$$
f(x, y) \cong f_{M_{\max }}(x, y)=\sum_{m=0}^{M_{\max }} \sum_{n=0}^{m} \lambda_{m-n, n} P_{m-n}(x) P_{n}(y)
$$

\section{Legendre Moments Computing}

In digital image processing, we can only observe an image function $f(x, y)$ at discrete pixels, so the discrete version of $f(x, y)$ becomes $f\left(x_{i}, y_{j}\right)$. In Legendre moment computing, it has been a common practice to apply (5) directly. However, when the order of Legendre moments increases, $\Delta x \Delta y$ in (5) can no longer produce an accurate result.

Figure 1 shows an example of the distribution of higher order Legendre polynomials within a pixel. It is obvious that the integration of $P_{100}(x) P_{100}(y)$ within this pixel is different from $\Delta x \Delta y$.

To improve the accuracy of Legendre moment computation, we approximate $\lambda_{m n}$ by:

$$
\hat{\lambda}_{m n}=\sum_{i=1}^{M} \sum_{j=1}^{N} f\left(x_{i}, y_{j}\right) h_{m n}\left(x_{i}, y_{j}\right)
$$

Where:

$$
h_{m n}\left(x_{i}, y_{j}\right)=\int_{x_{i}-\frac{\Delta x}{2}}^{x_{i}+\frac{\Delta x}{2}} \int_{y_{i}-\frac{\Delta y}{2}}^{y_{i}+\frac{\Delta y}{2}} P_{m}(x) P_{n}(y) d x d y
$$

By some well-known techniques of numerical integration, the integrations in (9) can be approximated with various accuracies. For example, the alternative extended Simpson's rule was applied to compute Legendre moments (Flannery, 1992; Liao and Pawlak, 1996).

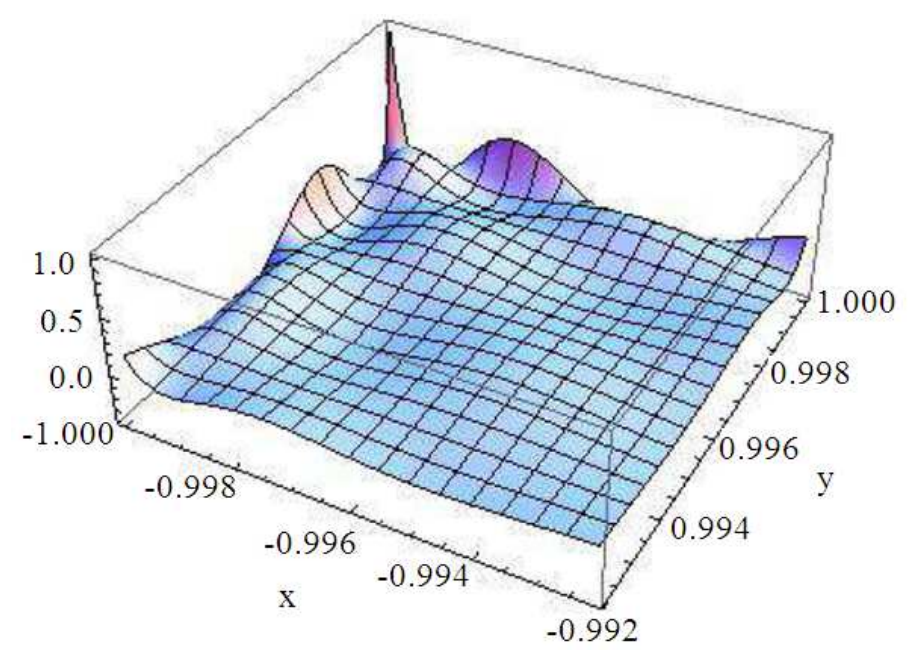

Fig. 1. The distribution of $P_{100}(x) P_{100}(y)$ in a corner pixel of an image size 
In this research, referring to a recent work (Wang and Liao, 2013), a straightforward $k \times k$ numerical scheme is utilized to calculate the double integrations in (9). By dividing a pixel into $k \times k$ sub regions with the same weights, we can reduce the computational errors of Legendre polynomials substantially.

\section{Image Reconstruction from Legendre Moments}

To verify our proposed solutions for more accurate Legendre moment computation, we would examine the image reconstruction determined by:

$$
\hat{f}_{M_{\max }}(x, y)=\sum_{m=0}^{M_{\max }} \sum_{n=0}^{m} \hat{\lambda}_{m-n, n} P_{m-n}(x) P_{n}(y)
$$

which is a newer version of (7) with the Legendre moments $\lambda_{m-n, n}$ replaced by their approximations given by (8). It is important to note that when the given highest Legendre moment order $M_{\max }$ is increased, the previously determined $\hat{\lambda}_{m-n, n}$ do not change.

Figure 2 shows two testing images utilized in this research. Each of the testing images is sized by $256 \times 256$ with 256 different gray levels.

To compare the reconstructed images with the original image, we have adopted the Peak Signal to Noise Ratio (PSNR) as the measurement, which is image independent and can be used to evaluate the reconstruction performance generally. PSNR is the ratio between the maximum power of the signal and the affecting noise and is defined as:

$$
P S N R=10 \log _{10}\left(\frac{G_{M a x}^{2}}{M S E}\right)
$$

where, $G_{M a x}$ is the maximum gray level of the image, which is 255 in our case and MSE is the Mean Square Error given by:

$$
M S E=\frac{1}{M N} \sum_{i=1}^{M} \sum_{j=1}^{N}\left[f\left(x_{i}, y_{j}\right)-\hat{f}\left(x_{i}, y_{j}\right)\right]^{2}
$$

We have conducted the image reconstructions from different maximum Legendre moment orders with various $k \times k$ numerical schemes. Figure 3 shows the plotting of PSNR values from the Legendre moment reconstruction performances on testing image Fig. 2a with different maximum orders and $k \times k$ numerical schemes, while Table 1 displays some PSNR values presented in Fig. 3.

Table 2 has shown some selected PSNR values from image reconstruction performances on testing image Fig. $2 \mathrm{~b}$ with the $11 \times 11$ numerical scheme and different maximum Legendre moment orders.

Figure 4 demonstrates some images reconstructed from Fig. 2a with various $k \times k$ numerical schemes and different maximum Legendre moment orders. It can be observed that the images reconstructed from higher orders of Legendre moments with $7 \times 7$ and $11 \times 11$ numerical schemes are very close to the original Fig. 2a visually.

Figure 5 presents some reconstructed images from Fig. $2 \mathrm{~b}$ with the $11 \times 11$ numerical scheme and different maximum Legendre moment orders.

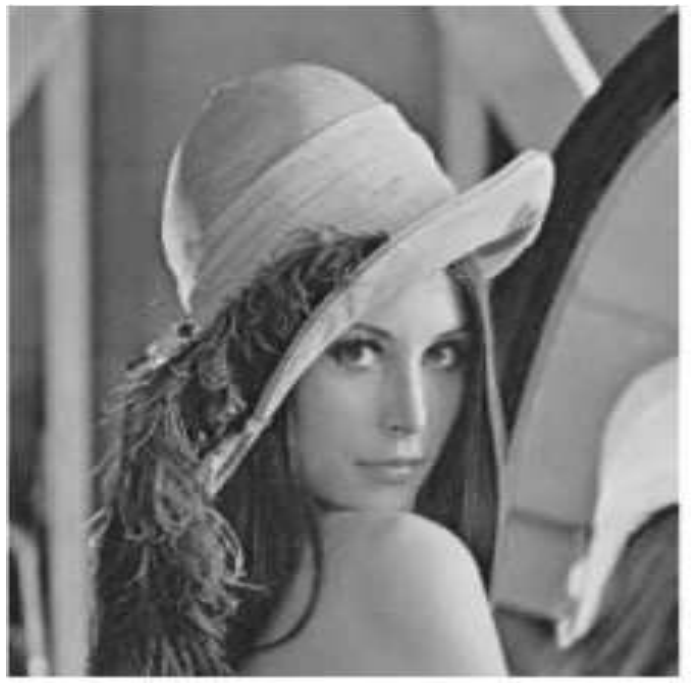

(a)

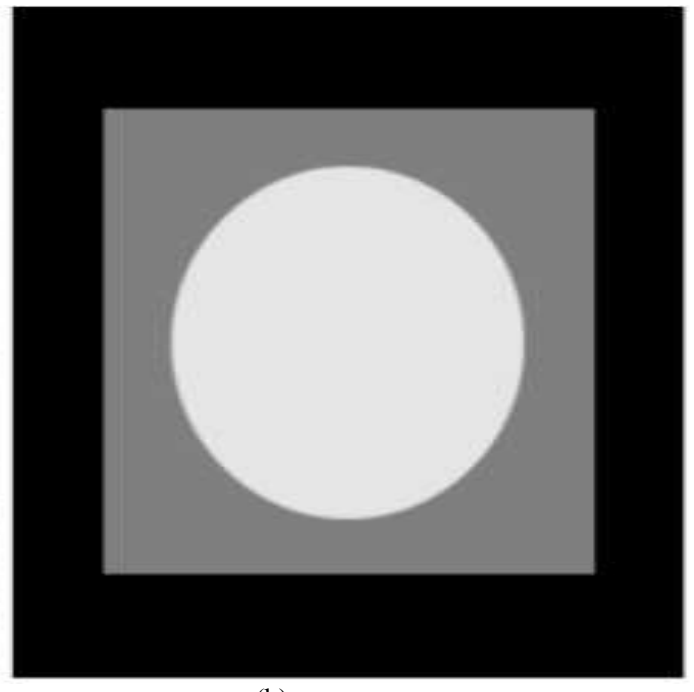

(b)

Fig. 2. Two testing images sized by $256 \times 256$ with 256 different gray levels 


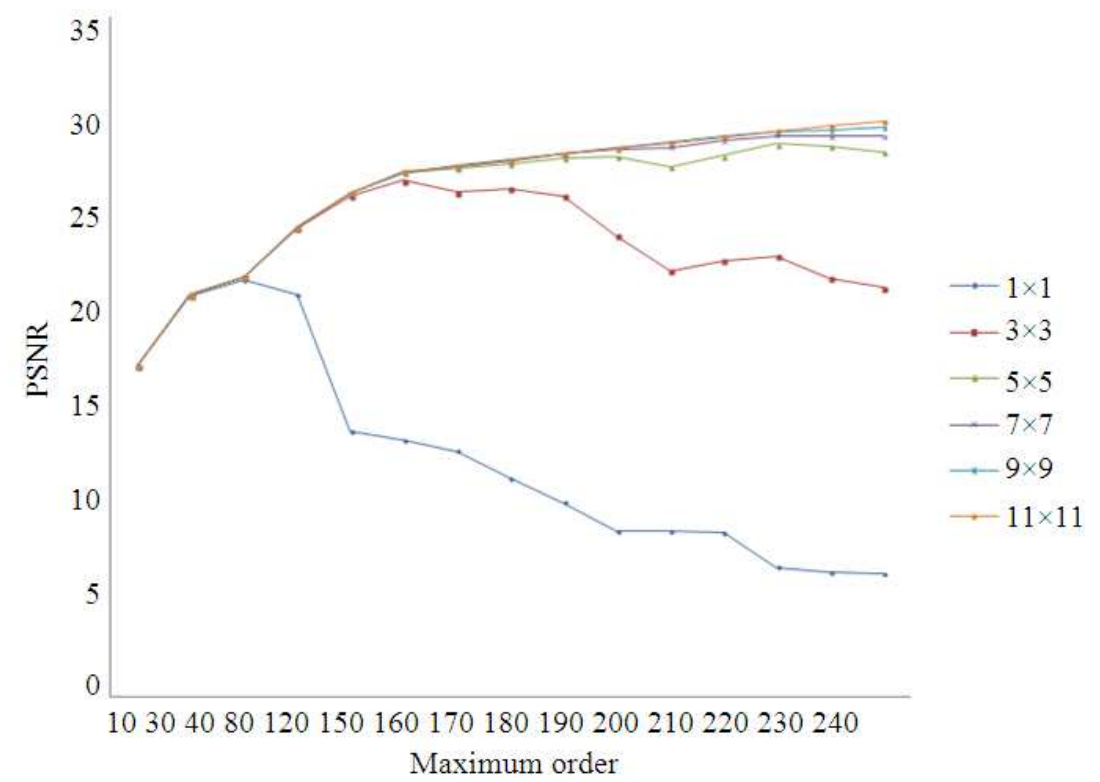

Fig. 3. PSNRs of the reconstructed Fig. 2a with different Legendre moment orders and $k \times k$ numerical schemes

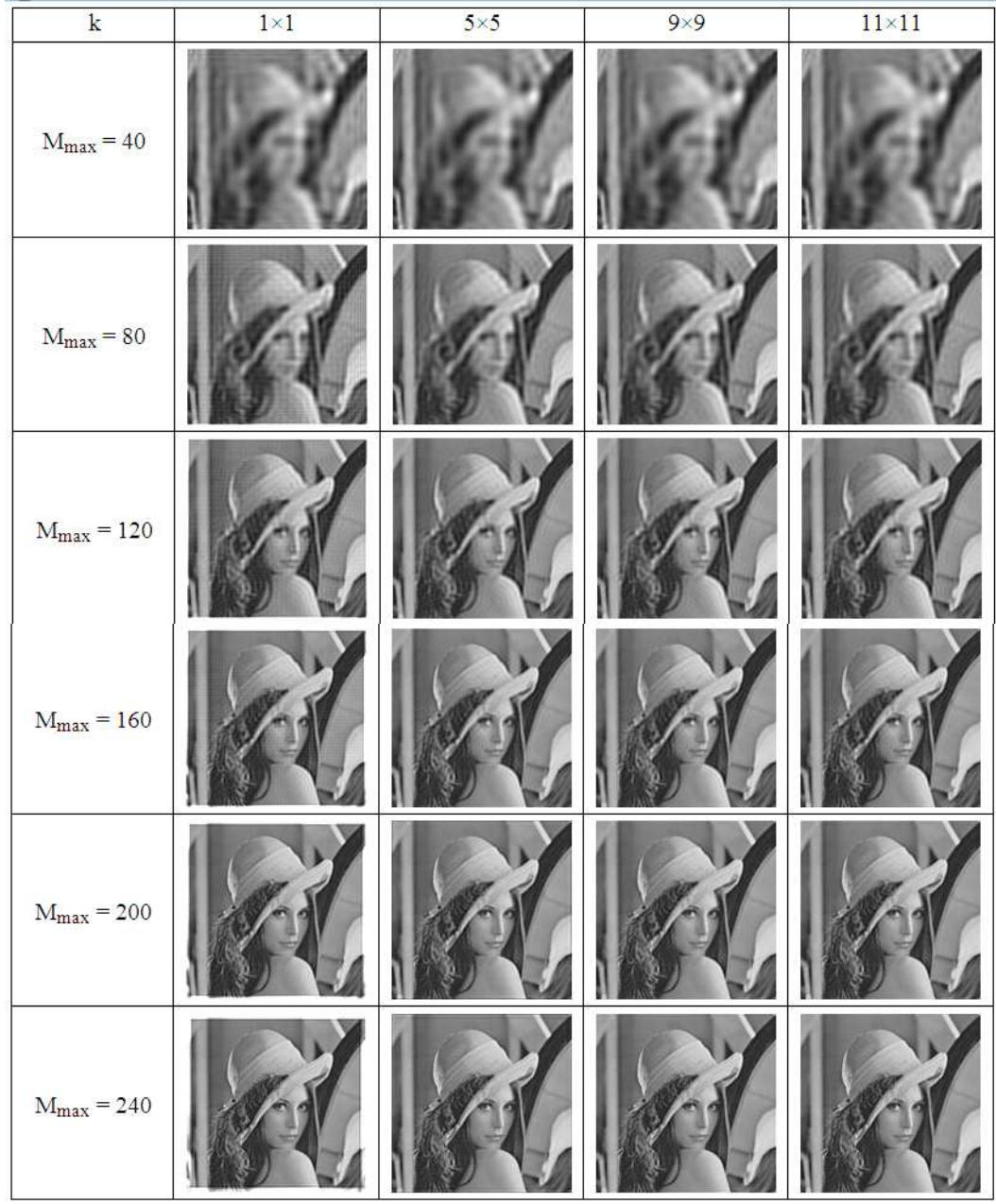

Fig. 4. Some reconstructed images from different Legendre moments orders with various $k \times k$ numerical schemes on Fig. 2a 


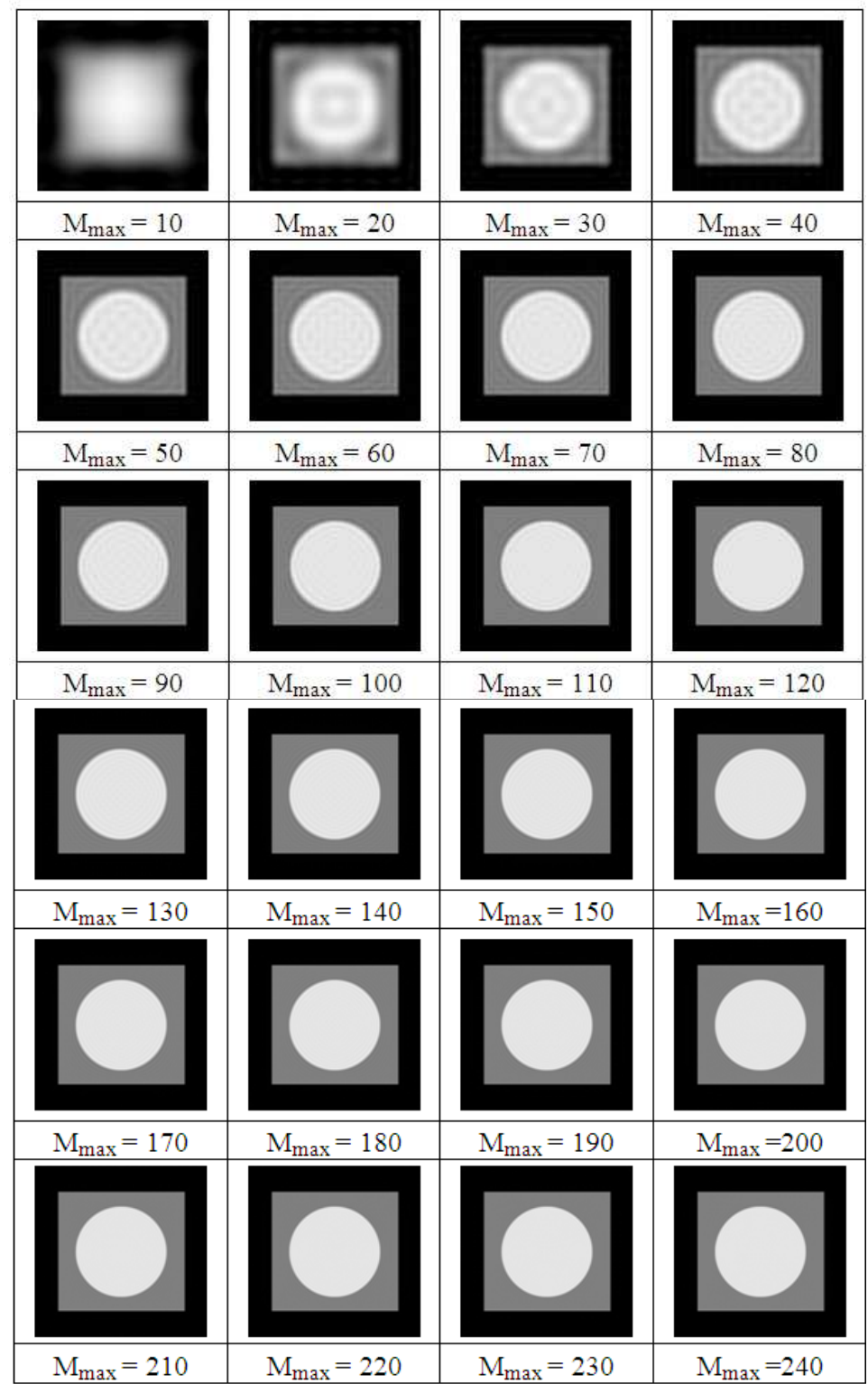

Fig. 5. Some reconstructed images from different Legendre moments orders with the $11 \times 11$ numerical scheme on Fig. $2 b$

\section{Image Reconstruction from a Partial Set of Legendre Moments}

According to the general moment theory, for a digital image, the lower order moments represent its fundamental features while the higher order moments characterize its details. Based on the previously presented improved moment computation techniques and precisely reconstructed images, we are able to investigate the individual contributions by a partial set of Legendre moments.

To examine the image reconstruction determined by a limited band of Legendre moments, we adopt the formula:

$$
\hat{f}_{\text {band }}(x, y)=\sum_{m=M_{\text {min }}}^{M_{\max }} \sum_{n=0}^{m} \hat{\lambda}_{m-n, n} P_{m-n}(x) P_{n}(y)
$$

To perform the image reconstructions. In (13), $M_{\max }$ and $\mathrm{M}_{\min }$ denote the highest and lowest Legendre moments involved in image reconstructions. The same two testing images shown in Fig. 2 are utilized here.

The sub-figures (a) to (d) of Fig. 6 show the images of the reconstructed Fig. 2a from partial sets of Legendre moments of orders 0 to 40,41 to 80,81 to 120 and 121 to 200 , respectively. The numerical scheme $k=11$ is adopted to compute Legendre 
moments in this experiment. Figure 6e presents the reconstructed image of orders 0 to 200 and Fig. $6 f$ displays the result of direct addition operation of images Fig. 6a-d. All sub-figures illustrated in Fig. 6 are the direct reconstructed results without any image enhancement for displaying. As expected, the images presented in Fig. 6e and $6 \mathrm{f}$ are identical.

The results shown in Fig. 6 have clarified the general moment theory that the lower order Legendre moments represent fundamental features of an image, while the higher order Legendre moments describe its details.

To address the issue of image reconstruction from a partial set of Legendre moments further, we have conducted additional investigation on the testing image Fig. 2b.

Figure 7 displays some reconstructed images from using lower orders of individual Legendre moments, $51,52,53,54,55$ and the set of 51 to 55 , respectively. The histograms of those reconstructed images show that all of the image pixels have gray level values of 25 or less. For a better displaying of image details, we have re-scaled these images by a multiplier of 10 .

Figure 8 shows some images reconstructed by using higher orders of individual Legendre moments, 196, 197, 198, 199, 200 and the set of 196 to 200, respectively. Since the highest gray level of all images is 9 , a multiplier of 25 is applied for displaying.
From the experimental results shown in Fig. 7 and 8 , we have some observations about the characteristics of individual orders of Legendre moments in image representing. Firstly, each individual order of moments represent the unique image details independently. Secondly, the even orders of Legendre moments describe more image details than the odd orders of Legendre moments. To emphasize this discovery, we have conducted the images reconstructions from all even order, odd orders and all orders of Legendre moments between 0 and 240 and displayed the results in Fig. 9a-c, respectively.

We can observe that Fig. 9b has presented very limited information from the original testing image Fig. 2b. On the other hand, the PSNR value between Fig. $9 \mathrm{a}$ and $9 \mathrm{c}$ is 39.219 , which indicates that the similarity of an image reconstructed from even orders and that of all orders of Legendre moments is very high.

To address this interesting characteristic of Legendre moments further, we have conducted the image reconstructions from the even orders of Legendre moments only. Figure 10 presents some reconstructed Fig. $2 b$ from the even orders of Legendre moments. Compared with each of the sub-figures displayed in Fig. 5 , the two sets of reconstructed images appear very similar visually. Figure 11 shows the PSNR values calculated from the image reconstructions displayed in both Fig. 5 and 10, respectively.

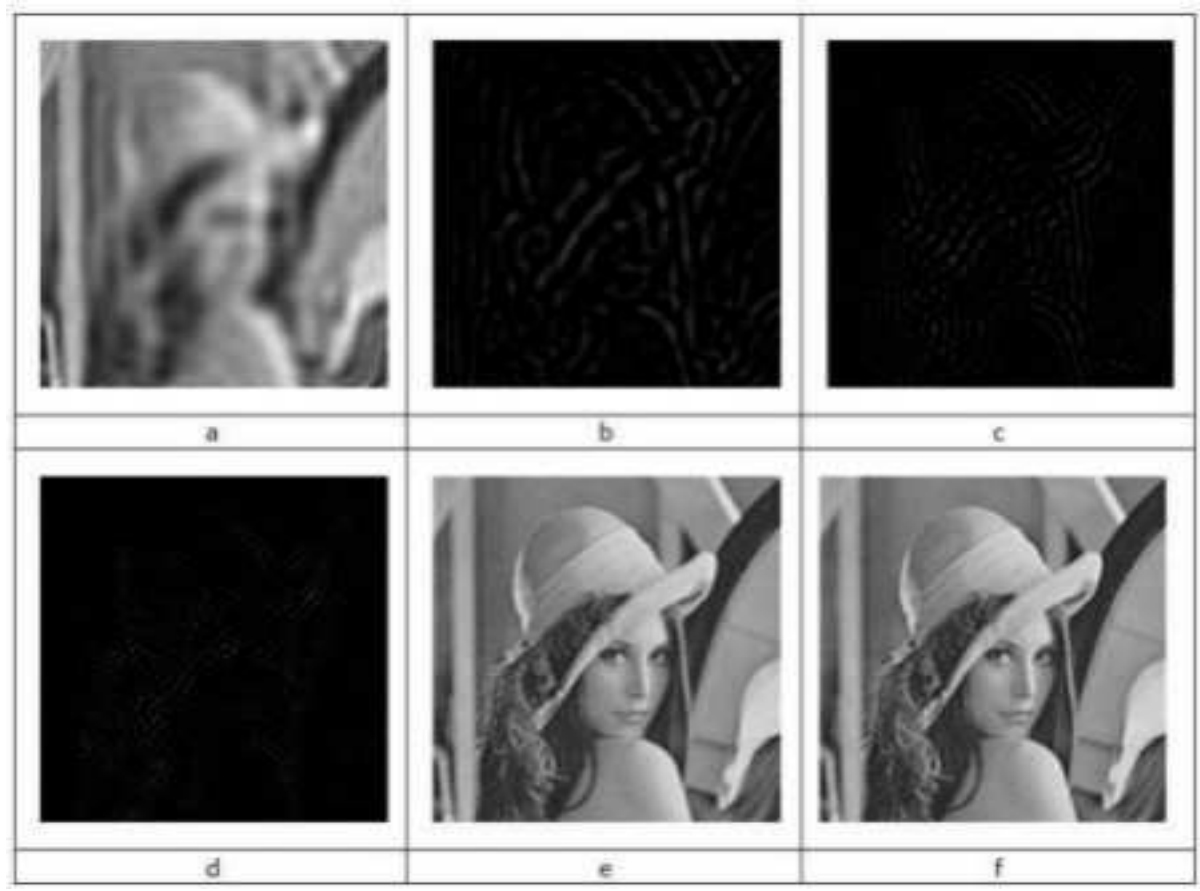

Fig. 6. Sub-figures (a) to (f) are the reconstructed images of Fig. 2 a from Legendre moments of orders 0 to 40,41 to 80,81 to 120, 121 to 200, 0 to 200 and the direct addition operation of images (a) to (d), respectively 


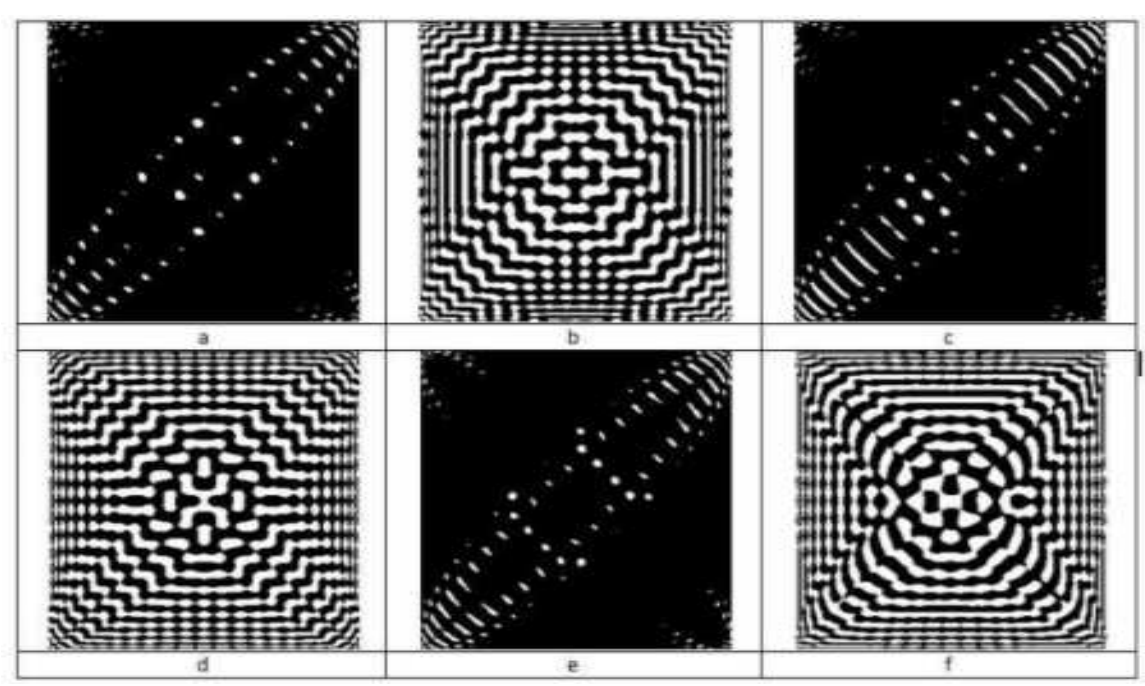

Fig. 7. Sub-figures (a) to (f) are the reconstructed images of Fig. $2 b$, applying the $11 \times 11$ numerical scheme, from Legendre moments of orders $51,52,53,54,55$ and 51 to 55 , respectively. The gray levels of all image pixels are multiplied by 10

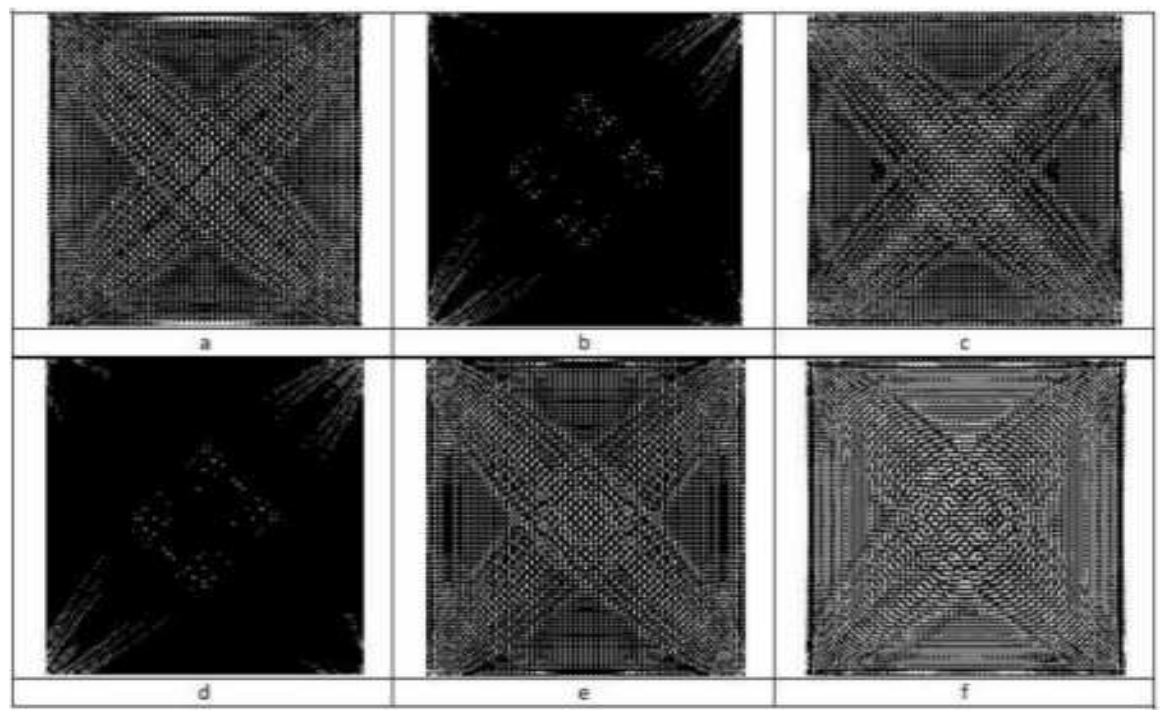

Fig. 8. Sub-figures (a) to (f) are the reconstructed images of Fig. $2 b$, applying the $11 \times 11$ numerical scheme, from Legendre moments of orders 196, 197, 198, 199, 200 and 196 to 200, respectively. The gray levels of all image pixels are multiplied by 25

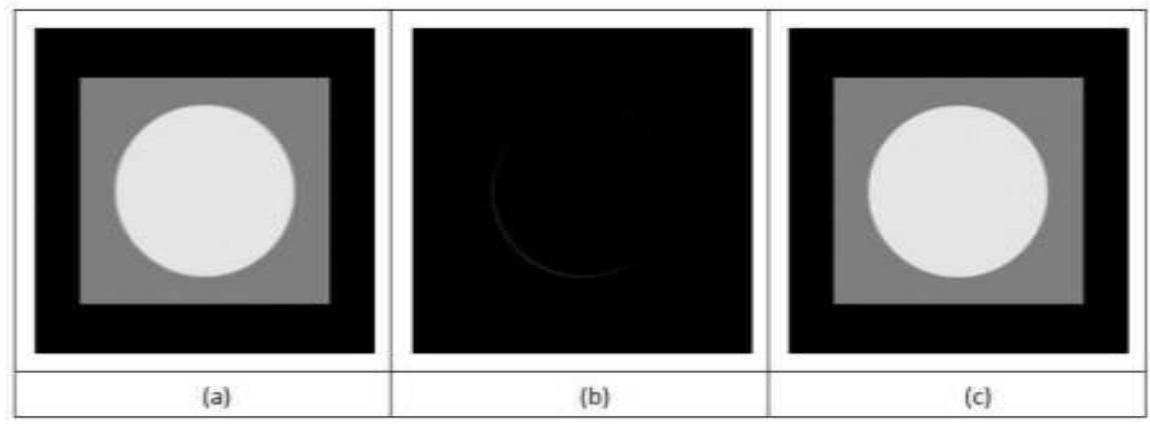

Fig. 9. Images reconstructed from all even number orders 0 to 240 , all odd number orders from 1 to 239 and all orders from 0 to 240 , respectively 


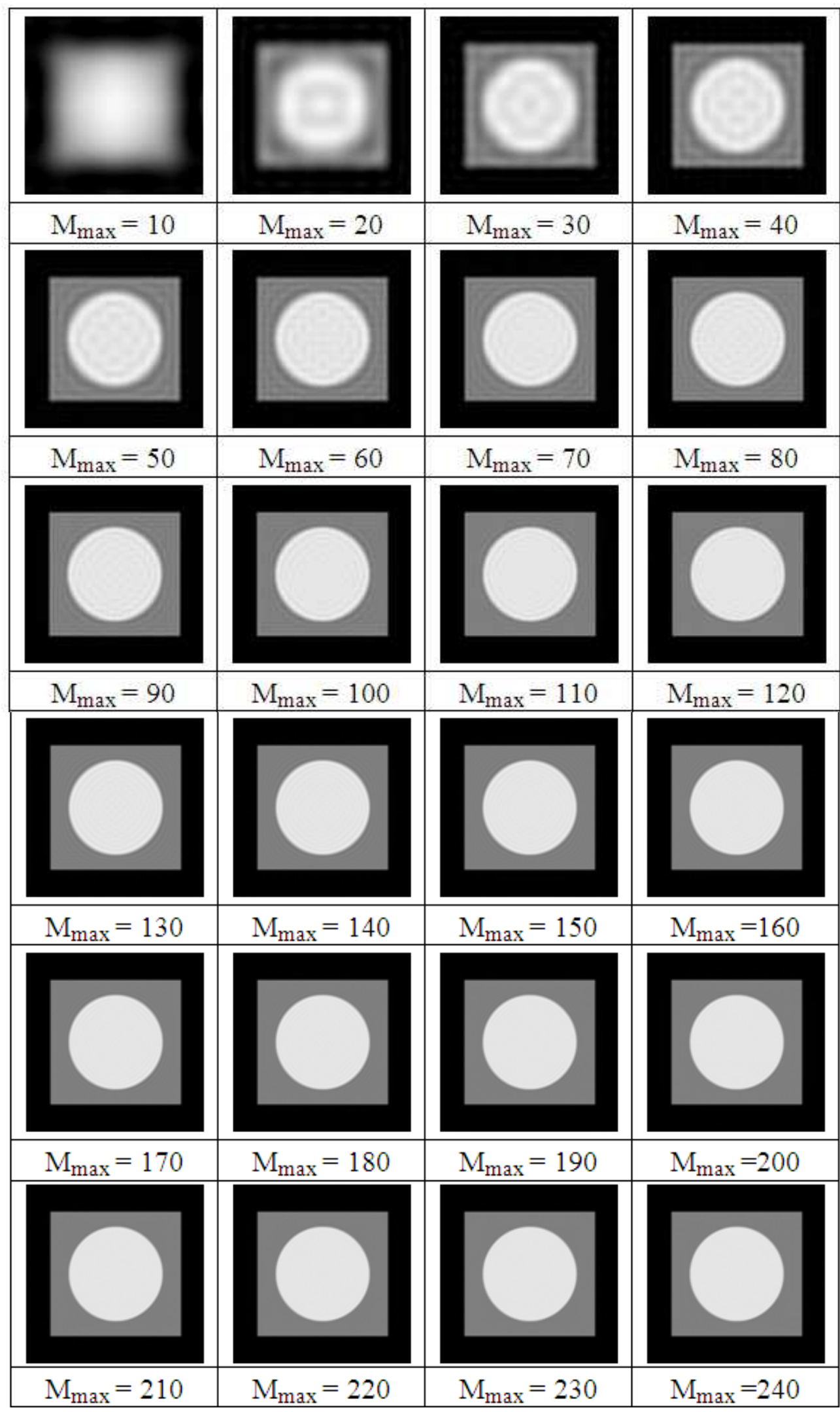

Fig. 10. Image reconstructed from different even orders of Legendre moments with the $11 \times 11$ numerical scheme on Fig. $2 b$ 


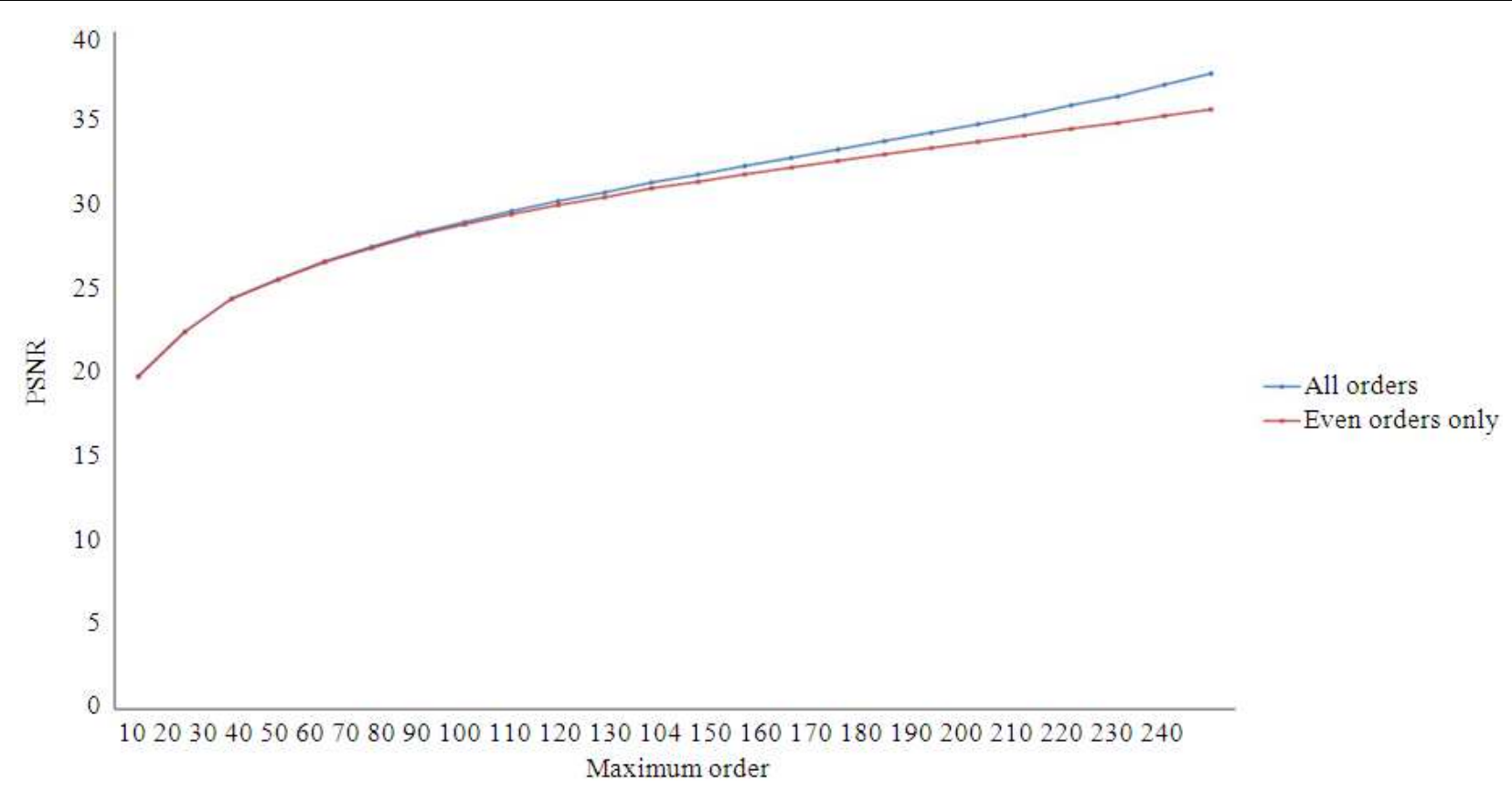

Fig. 11. PSNRs of the reconstructed Fig. 2b displayed in Fig. 5 and 10, respectively

Table 1. PSNR values of different $k \times k$ numerical schemes for reconstruction performances on Fig. $2 \mathrm{a}$

\begin{tabular}{lcccccrrr}
\hline Order $\left(\mathrm{M}_{\max }\right)$ & 10 & 40 & 80 & 120 & 150 & 180 & 210 & 240 \\
\hline $1 \times 1$ & 16.987 & 21.460 & 20.681 & 13.676 & 13.184 & 9.994 & 8.455 & 6.331 \\
$3 \times 3$ & 16.987 & 21.591 & 24.098 & 25.798 & 26.575 & 25.748 & 22.438 & 21.042 \\
$5 \times 5$ & 16.987 & 21.593 & 24.144 & 25.902 & 26.961 & 27.743 & 27.845 & 28.055 \\
$7 \times 7$ & 16.987 & 21.593 & 24.154 & 25.922 & 27.006 & 27.934 & 28.609 & 28.833 \\
$9 \times 9$ & 16.987 & 21.593 & 24.156 & 25.942 & 27.008 & 27.927 & 28.839 & 29.283 \\
$11 \times 11$ & 16.987 & 21.593 & 24.157 & 25.952 & 27.013 & 27.931 & 28.822 & 29.637 \\
\hline
\end{tabular}

Table 2. PSNR values of the $11 \times 11$ numerical scheme for reconstruction performances on Fig. $2 \mathrm{~b}$

\begin{tabular}{|c|c|c|c|c|c|c|c|c|}
\hline $\operatorname{Order}\left(\mathrm{M}_{\max }\right)$ & 10 & 40 & 80 & 120 & 150 & 180 & 210 & 240 \\
\hline $11 \times 11$ & 19.641 & 25.384 & 28.759 & 31.095 & 32.555 & 34.037 & 35.651 & 37.530 \\
\hline
\end{tabular}

\section{Concluding Remarks}

In this research, an innovate attempt to improve the computational accuracy of Legendre moments is proposed. To examine the more accurately computed Legendre moments, we have performed image reconstructions from higher orders of Legendre moments with satisfactory results.

By conducting image reconstructions from a partial set of Legendre moments, we have clarified that the lower orders of moments mainly contain the fundamental information of the original image, while the higher orders moments preserve the detailed image information. We have concluded that each individual finite set of Legendre moments will represent the unique image details independently. We have also discovered that the even orders of Legendre moments describe most of image characteristics, while the odd orders of Legendre moments would only present very limited information from the original image.

\section{Acknowledgment}

The researchers wish to thank the reviewers for their helpful comments. This author is supported by NSERC Discovery Grant.

\section{Author's Contributions}

All authors equally contributed in this work.

\section{Ethics}

This article is original and contains unpublished material. The corresponding author confirms that all of the other authors have read and approved the manuscript and no ethical issues involved. 


\section{References}

Courant, R. and D. Hilbert, 1966. Methods of Mathematical Physics. 1st Edn., Wiley.

Flannery, B.P., 1992. Saul A Teukolsky and William Vetterling. Numerical recipes in c. Press Syndicate of the University of Cambridge, William H Press, New York.

Flusser, J., T. Suk and B. Zitova, 2009. Moments and moment invariants in pattern recognition. 1st Edn., John Wiley and Sons, Chichester, West Sussex, ISBN-10: 0470684763, pp: 312.

Hu, M.K., 1962. Visual pattern recognition by moment invariants. IRE Trans. Inform. Theory, 8: 179-187. DOI: 10.1109/TIT.1962.1057692

Liao, S.X. and M. Pawlak, 1996. On image analysis by moments. IEEE Trans. Pattern Anal. Mach. Intelli., 18: 254-266. DOI: 10.1109/34.485554

Mukundan, R. and K.R. Ramakrishnan, 1998. Moment Functions in Image Analysis-Theory and Applications. 1st Edn., Illustrated, World Scientific, Singapore, ISBN-10: 9810235240, pp: 150.
Pawlak, M., 2006. Image Analysis by Moments: Reconstruction and Computational Aspects. 1st Edn., Illustrated, Oficyna Wydawnicza Politechniki Wrocławskiej, Wrocław, ISBN-10: 8370859666, pp: 217.

Sansone, G., 1991. Orthogonal Functions. 1st Edn., Dover Publications.

Teague, M.R., 1980. Image analysis via the general theory of moments. Opt. Soc. Am., 70: 920-930. DOI: $10.1364 /$ JOSA.70.000920

Teh, C.H. and R.T. Chin, 1988. On image analysis by the methods of moments. IEEE Trans. Patt. Anal. Mach. Intell., 10: 496-513. DOI: 10.1109/34.3913

Wang, X. and S. Liao, 2013. Image Reconstruction from Orthogonal Fourier-Mellin Moments. In: Image Analysis and Recognition. Kamel, M. and A. Campilho (Eds.)., Springer Berlin Heidelberg, ISBN-10: 978-3-642-39093-7, pp: 687-694. 